\title{
A CLASS OF LIMIT THEOREMS
}

\author{
BY \\ M. $\mathrm{KAC}^{(1)}$
}

1. Introduction. In a recent paper [1] D. A. Darling and the present author studied the asymptotic distributions of occupation times for general Markoff processes. The limit theorems obtained in $[1\rfloor$ depended on the "infinite part" of the Laplace transform (or the generating function when time is discretized) of the transition probabilities.

In the present paper a class of limit theorems which depend on the "finite part" are discussed. The methods form a simplified version of methods used by the author in the study of certain potential theoretic questions. These potential theoretic questions will be discussed in a subsequent publication. In the sequel the simplest case will be studied and the principal results are to be found in formulas (7.6a) and (7.6b).

We consider a sequence of independent, identically distributed random variables $X_{1}, X_{2}, \cdots$ whose density function $\rho(x)$ is even and whose characteristic function $\phi(\xi)$ is integrable in $(-\infty, \infty)$ and such that

$$
\phi(\xi) \sim 1-|\xi|^{\gamma}, \quad \xi \rightarrow 0,1 \leqq \gamma \leqq 2 .
$$

Let $\Omega$ be a bounded measurable set and denote by $P_{n}(l ; \Omega)$ the probability that exactly $l$ among the first $n$ partial sums $s_{k}=X_{1}+X_{2}+\cdots+X_{k}$ fall within $\Omega$. Formulas (7.6a) and (7.6b) assert that the limits

$$
\lim _{n \rightarrow \infty} n^{1-1 / \gamma} P_{n}(l ; \Omega),
$$$$
1<\gamma \leqq 2 \text {, }
$$

and

$$
\lim _{n \rightarrow \infty} \log n P_{n}(l ; \Omega),
$$$$
\gamma=1,
$$

exist. The numerical determination of these limits is somewhat involved and they are expressible in terms of the eigenvalues and eigenfunctions of the integral equation

$$
\int_{\Omega} L(x-y) \psi(y) d y=\lambda \psi(x), \quad x \in \Omega
$$

where

Received by the editors May 19, 1956.

(1) This research was supported by the United States Air Force under Contract No. AF 18(600)-685 monitored by the Office of Scientific Research. 


$$
L(x)=\frac{1}{2 \pi} \int_{-\infty}^{\infty} \frac{1-\cos x \xi}{1-\phi(\xi)} \phi(\xi) d \xi .
$$

Analogous results can be obtained for random variables having the lattice structure (see $\$ 8$ ). $-L(x)$ is precisely the "finite part" of the generating function of transition probabilities (see (4.6)).

2. The "forward" equation. Let $V(x)$ be the characteristic function of the set $\Omega$ and consider

$$
E\left\{\exp -u \sum_{1}^{n} V\left(s_{k}\right)\right\}
$$

Set

$$
\begin{aligned}
Q_{n}(x)=\int_{-\infty}^{\infty} \cdots \int_{-\infty}^{\infty} \exp \left[-u \sum_{1}^{n} V\left(\xi_{k}\right)\right] \rho\left(\xi_{1}\right) \rho\left(\xi_{2}-\xi_{1}\right) \cdots \\
\rho\left(\xi_{n}-\xi_{n-1}\right) \rho\left(x-\xi_{n}\right) d \xi_{1} \cdots d \xi_{n}
\end{aligned}
$$

and observe that

$$
\int_{-\infty}^{\infty} Q_{n}(x) d x=E\left\{\exp -u \sum_{1}^{n} V\left(s_{k}\right)\right\}
$$

The $Q_{n}$ 's obey the relation

$$
Q_{n}(x)=\int_{-\infty}^{\infty} e^{-u V(\xi)} Q_{n-1}(\xi) \rho(x-\xi) d \xi,
$$

where

$$
Q_{0}(\xi)=\rho(\xi)
$$

and consequently setting

$$
R(x)=\sum_{n=0}^{\infty} Q_{n}(x) z^{n}, \quad 0<z<1
$$

we obtain

$$
R(x)=\rho(x)+z \int_{-\infty}^{\infty} R(\xi) e^{-u V(\xi)} \rho(x-\xi) d \xi .
$$

It is clear that $R$ depends also on $u$ and $z$ but we suppress this dependence to simplify the notation.

Let now

$$
o^{(n)}(x)=\rho * \rho * \rho \cdots * \rho
$$

i.e., 


$$
\rho^{(n)}(x)=\frac{1}{2 \pi} \int_{-\infty}^{\infty} e^{-i \xi x} \phi^{n}(\xi) d \xi,
$$

where $\phi(\xi)$ is the characteristic function of $\rho(x)$ i.e.,

$$
\phi(\xi)=\int_{-\infty}^{\infty} \rho(x) e^{i \xi x} d x .
$$

We shall assume that $\phi$ is absolutely integrable in $(-\infty, \infty)$ and real (so that $\rho(x)$ is continuous and even). Multiplying both sides of (2.6) by $\rho^{(n)}(\eta-x)$ and integrating over $x$ we obtain

$$
\int_{-\infty}^{\infty} R(x) \rho^{(n)}(\eta-x) d x=\rho^{(n+1)}(\eta)+z \int_{-\infty}^{\infty} R(\xi) e^{-u V(\xi)} \rho^{(n+1)}(\eta-\xi) d \xi .
$$

Introducing the function

$$
K_{z}(x)=\sum_{n=1}^{\infty} z^{n-1} \rho^{(n)}(x)
$$

we obtain from (2.9)

$$
\begin{aligned}
z \int_{-\infty}^{\infty} R(x) K_{z}(\eta-x) d x & \\
& =K_{z}(\eta)-\rho(\eta)+z \int_{-\infty}^{\infty} R(\xi) e^{-u V(x)}\left[K_{z}(\eta-\xi)-\rho(\eta-\xi)\right] d \xi
\end{aligned}
$$

which, using (2.6), can easily be reduced to the form

$$
R(\eta)=K_{z}(\eta)-z\left(1-e^{-u}\right) \int_{\Omega} R(\xi) K_{z}(\eta-\xi) d \xi .
$$

This is the "forward" equation.

3. The "backward" equation. Let now

$$
\begin{array}{r}
P_{n}(y)=\int_{-\infty}^{\infty} \cdots \int_{-\infty}^{\infty} \exp \left[-u \sum_{1}^{n} V\left(\xi_{k}\right)\right] \rho\left(\xi_{1}-y\right) \rho\left(\xi_{2}-\xi_{1}\right) \cdots \\
\rho\left(\xi_{n}-\xi_{n-1}\right) d \xi_{1} \cdots d \xi_{n}
\end{array}
$$

and observe that

$$
E\left\{\exp \left(-u \sum_{1}^{n} V\left(s_{k}\right)\right)\right\}=P_{n}(0) .
$$

The $P_{n}$ 's satisfy the relation

$$
P_{n}(y)=\int_{-\infty}^{\infty} P_{n-1}(\xi) e^{-u V(\xi)} \rho(\xi-y) d \xi
$$


which for even $\rho$ is identical with (2.4a) (we have assumed that $\rho$ is even). However the initial condition is quite different and, in fact,

$$
P_{0}(\xi) \equiv 1 \text {. }
$$

Setting

$$
S(x)=\sum_{n=0}^{\infty} P_{n}(x) z^{n}
$$

we obtain, repeating step by step the derivation of $\S 2$,

$$
S(\eta)=\frac{1}{1-z}-z\left(1-e^{-u}\right) \int_{\Omega} S(\xi) K_{\mathbf{z}}(\eta-\xi) d \xi
$$

which is the "backward" equation.

4. The limit $z \rightarrow 1$. We have from (2.8), (2.10) and the assumption that $\phi$ is absolutely integrable

$$
K_{z}(x)=\frac{1}{2 \pi} \int_{-\infty}^{\infty} \frac{e^{-i \xi x} \phi(\xi)}{1-z \phi(\xi)} d \xi .
$$

Since $\phi$ is even it follows easily that

$$
\lim _{\xi \rightarrow 0} \frac{1-\phi(\xi)}{\xi^{2}}=\int_{-\infty}^{\infty} x^{2} \rho(x) d x>0,
$$

where the second moment may be infinite.

Thus

$$
\frac{\xi^{2}}{1-\phi(\xi)}
$$

is bounded (and hence integrable) near $\xi=0$ and we can write

$$
\begin{aligned}
K_{z}(x)= & \frac{1}{2 \pi} \int_{-\infty}^{\infty} \frac{\phi(\xi)}{1-z \phi(\xi)} d \xi \\
& -\frac{1}{2 \pi} \int_{-\infty}^{\infty} \frac{(1-\cos x \xi)}{1-\phi(\xi)} \phi(\xi) d \xi+o(1-z) .
\end{aligned}
$$

Setting

$$
h(z)=\frac{1}{2 \pi} \int_{-\infty}^{\infty} \frac{\phi(\xi)}{1-z \phi(\xi)} d \xi
$$

and

$$
L(x)=\frac{1}{2 \pi} \int_{-\infty}^{\infty} \frac{(1-\cos \xi x)}{1-\phi(\xi)} \phi(\xi) d \xi
$$


we can write the decomposition (4.3) in the form

$$
K_{z}(x)=h(z)-L(x)+o(1-z) .
$$

It also follows that

$$
K_{z}(x)-h(z)
$$

is bounded on every bounded set of $x$ 's (although not uniformly bounded in $(-\infty, \infty))$. We shall assume now that

$$
h(z) \rightarrow \infty, \quad z \rightarrow 1 .
$$

Returning to (2.11) we rewrite it in the equivalent form

$$
R(\eta)=\left(K_{z}(\eta)-h(z)\right)-z\left(1-e^{-u}\right) \int_{\Omega} R(\xi)\left(K_{z}(\eta-\xi)-h(z)\right) d \xi
$$

$$
+h(z)\left(1-z\left(1-e^{-u}\right) \int_{\Omega} R(\xi) d \xi\right) .
$$

Integrating (2.11) with respect to $\eta$ from $-\infty$ to $\infty$ and using the trivial identity

$$
\int_{-\infty}^{\infty} K_{z}(\eta) d \eta=\frac{1}{1-z}
$$

we obtain

$$
1-z\left(1-e^{-u}\right) \int_{\Omega} R(\xi) d \xi=(1-z) \int_{-\infty}^{\infty} R(\eta) d \eta>0
$$

and hence

$$
\int_{\Omega} R(\xi) d \xi<\frac{1}{z\left(1-e^{-u}\right)} .
$$

Integrating (4.8) with respect to $\eta$ over $\Omega$ we get (denoting by $|\Omega|$ the measure of $\Omega$ )

$$
\begin{aligned}
|\Omega| h(z) & \left(1-z\left(1-e^{-u}\right) \int_{\Omega} R(\xi) d \xi\right) \\
= & \int_{\Omega} R(\eta) d \eta-\int_{\Omega}\left(K_{z}(\eta)-h(z)\right) d \eta \\
& \quad+z\left(1-e^{-u}\right) \int_{\Omega} R(\xi) \int_{\Omega}\left(K_{z}(\eta-\xi)-h(z)\right) d \eta d \xi
\end{aligned}
$$

From (4.10) and the fact that $K_{z}(x)-h(z)$ is bounded over every bounded set of $x$ 's it follows immediately that 


$$
h(z)\left(1-z\left(1-e^{-u}\right) \int_{\Omega} R(\xi) d \xi\right)
$$

remains bounded as $z \rightarrow 1$. This in conjunction with (4.8) implies that $R(\eta)$ remains bounded (over every bounded set of $\eta$ 's) as $z \rightarrow 1$.

Since $R(\eta)$ is an increasing function of $z$ it follows that

$$
\lim _{z \rightarrow 1} R(\eta)=T(\eta)
$$

exists for every $\eta$. Finally, (4.8) and (4.12) imply (using the theorem on bounded convergence) that

$$
\left.\lim _{z \rightarrow 1} h(z)\left(1-z\left(1-e^{-u}\right) \int_{\Omega} R(\xi) d \xi\right)\right)=G(u)
$$

exists and moreover

$$
T(\eta)=-L(\eta)+\left(1-e^{-u}\right) \int_{\Omega} T(\xi) L(\eta-\xi) d \xi+G(u) .
$$

Since $h(z) \rightarrow \infty$ as $z \rightarrow 1$ it follows from (4.13) that

$$
\left(1-e^{-u}\right) \int_{\Omega} T(\xi) d \xi=1
$$

and hence from (4.9) and (4.13)

$$
\int_{-\infty}^{\infty} R(\eta) d \eta \sim \frac{G(u)}{(1-z) h(z)},
$$

or in other words

$$
\sum_{n=0}^{\infty} z^{n} E\left\{\exp \left[-u \sum_{1}^{n} V\left(s_{k}\right)\right]\right\} \sim \frac{G(u)}{(1-z) h(z)}, \quad z \rightarrow 1 .
$$

5. The limit $z \rightarrow 1$ for the "backward" equation. We first note that

$$
S(0)=\int_{-\infty}^{\infty} R(\eta) d \eta \sim \frac{G(u)}{(1-z) h(z)}
$$

and since

$$
\begin{aligned}
P_{n}(y)=\int_{-\infty}^{\infty} \cdots \int_{-\infty}^{\infty} \exp \left[-u \sum_{1}^{n} V\left(y+\xi_{k}\right)\right] \rho\left(\xi_{1}\right) \rho\left(\xi_{2}-\xi_{1}\right) \cdots \\
\\
\quad \rho\left(\xi_{n}-\xi_{n-1}\right) d \xi_{1} \cdots d \xi_{n}
\end{aligned}
$$

we see that $P_{n}(y)$ is related to the set $\Omega-y$ (translation of $\Omega$ by $-y$ ) in the same way as $P_{n}(0)$ is related to the set $\Omega$. 
It thus follows that

$$
S(y) \sim \frac{G(u ; y)}{(1-z) h(z)}
$$

and moreover it can easily be shown that

$$
(1-z) h(z) S(y)
$$

remains bounded as $z \rightarrow 1$ over every bounded set of $y$ 's.

Summarizing the above we can say that

$$
\lim _{z \rightarrow 1}(1-z) h(z) S(y)=G(u ; y)
$$

exists and that the theorem on bounded convergence is applicable to the functions $(1-z) h(z) S(y)$. We now rewrite (3.5) in the equivalent form

$$
\begin{aligned}
(1-z) h(z) S(\eta)= & h(z)\left(1-z\left(1-e^{-u}\right)(1-z) h(z) \int_{\Omega} S(\xi) d \xi\right) \\
& -z\left(1-e^{-u}\right)(1-z) h(z) \int_{\Omega} S(\xi)\left(K_{z}(\eta-\xi)-h(z) d \xi .\right.
\end{aligned}
$$

From (5.1), the theorem on bounded convergence, and the boundedness of $K_{z}(\eta-\xi)-h(z)$ it follows that

$$
\lim _{z \rightarrow 1} h(z)\left(1-z\left(1-e^{-u}\right)(1-z) h(z) \int_{\Omega} S(\xi) d \xi\right)=p(u)
$$

exists and moreover

$$
G(u ; \eta)=p(u)+\left(1-e^{-u}\right) \int_{\Omega} G(u ; \xi) L(\eta-\xi) d \xi .
$$

Furthermore from (5.3) we infer that

$$
\left(1-e^{-u}\right) \int_{\Omega} G(u ; \xi) d \xi=1 .
$$

One can use (5.4) and (5.5) as well as (4.14) and (4.15) to determine $G(u)$. The advantage of (5.4) is that it is simpler.

6. Determination of $G(u)$. Consider the integral equation

$$
\int_{\Omega} L(\eta-\xi) \psi(\xi) d \xi=\lambda \psi(\eta), \quad \eta \in \Omega,
$$

and denote by $\lambda_{1}, \lambda_{2}, \ldots$ its eigenvalues and by $\psi_{1}, \psi_{2}, \cdots$ the corresponding normalized eigenfunctions.

From (5.4) we get 


$$
\left(1-\left(1-e^{-u}\right) \lambda_{j}\right) \int_{\Omega} G(u ; \eta) \psi_{j}(\eta) d \eta=p(u) \int_{\Omega} \psi_{j}(\eta) d \eta
$$

We shall prove now that at most one eigenvalue is greater than 1 . In fact, suppose that $\lambda_{i}>1$ and $\lambda_{j}>1$. We can choose constants $a$ and $b$ (not both 0 ) such that

$$
\int_{\Omega}\left(a \psi_{i}(x)+b \psi_{j}(x)\right) d x=0 .
$$

Set $\omega(x)=a \psi_{i}(x)+b \psi_{j}(x)$ and observe that

$$
\int_{\Omega} \int_{\Omega} \omega(x) K_{z}(x-y) \omega(y) d x d y+\frac{1}{z} \int_{\Omega} \omega^{2}(x) d x \geqq 0 .
$$

Because of (6.3) we have

$$
\int_{\Omega} \int_{\Omega} \omega(x)\left(K_{2}(x-y)-h(z)\right) \omega(y) d x d y+\frac{1}{z} \int_{\Omega} \omega^{2}(x) d x \geqq 0
$$

and letting $z \rightarrow 1$ we obtain

$$
-\int_{\Omega} \int_{\Omega} \omega(x) L(x-y) \omega(y) d x d y+\int_{\Omega} \omega^{2}(x) d x \geqq 0
$$

or

$$
\lambda_{i} a^{2}+\lambda_{j} b^{2} \leqq a^{2}+b^{2}
$$

contrary to the assumption that $\lambda_{i}>1, \lambda_{j}>1$. The same argument gives a stronger result if $\phi(\xi) \geqq 0$ i.e., $K_{z}(x-y)$ is positive definite. In fact, we have, instead of (6.4)

$$
\int_{\Omega} \int_{\Omega} \omega(x) K_{z}(x-y) \omega(y) d x d y \geqq 0
$$

and consequently at most one eigenvalue is positive. Since $L(0)=0$ at least one eigenvalue must be positive and hence exactly one eigenvalue is positive. To simplify the discussion we shall assume that 1 is not an eigenvalue i.e., $\lambda_{j} \neq 1$. From (6.2) and (5.5) one gets easily

$$
G(u)=G(u ; 0)=\frac{\frac{1}{1-e^{-u}}+\sum_{1}^{\infty} \frac{c_{j} l_{j}}{1-\left(1-e^{-u}\right) \lambda_{j}}}{\sum_{1}^{\infty} \frac{c_{j}^{2}}{1-\left(1-e^{-u}\right) \lambda_{j}}},
$$

where 


$$
c_{j}=\int_{\Omega} \psi_{j}(x) d x, \quad l_{j}=\int_{\Omega} L(\xi) \psi_{j}(\xi) d \xi .
$$

We can now write

$$
G(u)=\alpha_{0}+\alpha_{1} e^{-u}+\alpha_{2} e^{-2 u}+\cdots
$$

where

$$
\alpha_{0}=\left(1+\sum_{1}^{\infty} \frac{c_{j} l_{j}}{1-\lambda_{j}}\right) / \sum_{1}^{\infty} \frac{c_{j}^{2}}{1-\lambda_{j}}
$$

and the higher $\alpha$ 's are given by similar but more complicated formulas. We must still exclude the possibility that

$$
\sum_{1}^{\infty} \frac{c_{j}^{2}}{1-\lambda_{j}}=0
$$

This can be seen from the easily verified formula

$$
\frac{1}{1-e^{-u}}=\int_{\Omega} G(u ; \xi) d \xi=p(u) \sum_{1}^{\infty} \frac{c_{j}^{2}}{1-\left(1-e^{-u}\right) \lambda_{j}} .
$$

If (6.10) held it would follow that $p(u)$ is unbounded as $u \rightarrow \infty$. But from (5.4) we get

$$
\begin{aligned}
|p(u)||\Omega| & \leqq \int_{\Omega} G(u ; \eta) d \eta+\int_{\Omega} G(u ; \xi) \int_{\Omega} L(\eta-\xi) d \eta d \xi \\
& <M \int_{\Omega} G(u ; \eta) d \eta=\frac{M}{1-e^{-u}}
\end{aligned}
$$

and hence (6.10) cannot hold.

7. The limit theorems. We return now to formula (4.17)

$$
\sum_{n=0}^{\infty} z^{n} E\left\{\exp \left[-u \sum_{1}^{n} V\left(s_{k}\right)\right]\right\} \sim \frac{G(u)}{(1-z) h(z)}, \quad z \rightarrow 1 .
$$

In order to determine the behavior of

$$
E\left\{\exp \left[-u \sum_{1}^{n} V\left(s_{k}\right)\right]\right\}
$$

as $n \rightarrow \infty$ we need a Tauberian Theorem. For this we need an additional assumption on the characteristic function $\phi(\xi)$. We shall assume that for $\xi \rightarrow 0$

$$
\phi(\xi) \sim 1-|\xi|^{\gamma},
$$

$1 \leqq \gamma \leqq 2$,

which implies, in particular, that our random variables belong to the domain 
of attraction of the symmetric stable law of exponent $\gamma$. It is easily seen that (see e.g., [1])

$$
h(z) \sim \frac{1}{\gamma \sin (\pi / \gamma)} \frac{1}{(1-z)^{1-1 / \gamma}}, \quad 1<\gamma \leqq 2
$$

and

$$
h(z) \sim \frac{1}{\pi} \log \frac{1}{1-z},
$$$$
\gamma=1
$$

Under these circumstances the classical Tauberian Theorem can be applied and we get

$$
\sum_{n=1}^{N} E\left\{\exp \left[-u \sum_{1}^{n} V\left(s_{k}\right)\right]\right\} \sim \gamma \sin \frac{\pi}{\gamma} \frac{G(u)}{\Gamma(1 / \gamma+1)} N^{1 / \gamma}
$$

for $1<\gamma \leqq 2$, and

$$
\sum_{n=1}^{N} E\left\{\exp \left[-u \sum_{1}^{n} V\left(s_{k}\right)\right]\right\} \sim \pi G(u) \frac{N}{\log N}
$$

for $\gamma=1$. Since the sequence

$$
E\left\{\exp \left[-u \sum_{1}^{n} V\left(s_{k}\right)\right]\right\}
$$

is decreasing we obtain for (7.4a) and (7.4b)

(7.5a) $\quad \lim _{n \rightarrow \infty} n^{1-1 / \gamma} E\left\{\exp \left[-u \sum_{1}^{n} V\left(s_{k}\right)\right]\right\}=\frac{\sin \pi / \gamma}{\Gamma(1 / \gamma+1)} G(u)$,

for $1<\gamma \leqq 2$, and

$$
\lim _{n \rightarrow \infty} \log n E\left\{\exp \left[-u \sum_{1}^{n} V\left(s_{k}\right)\right]\right\}=\pi G(u)
$$

for $\gamma=1$.

It remains now to prove that

(7.6a) $\quad \lim _{n \rightarrow \infty} n^{1-1 / \gamma}$ Prob. $\left\{\sum_{1}^{n} V\left(s_{k}\right)=l\right\}=\frac{\sin \pi / \gamma}{\Gamma(1 / \gamma+1)} \alpha_{l}$,

for $1<\gamma \leqq 2$, and

$$
\lim _{n \rightarrow \infty} \log n \text { Prob. }\left\{\sum_{1}^{n} V\left(s_{k}\right)=l\right\}=\pi \alpha_{l},
$$

for $\gamma=1$. 
We carry out the proof of $(7.6 \mathrm{~b})$.

Let

$$
\log n \text { Prob. }\left\{\sum_{1}^{n} V\left(s_{k}\right)=l\right\}=a_{l}(n)
$$

and rewrite $(7.5 b)$ in the equivalent form

$$
\lim _{n \rightarrow \infty} \sum_{l=0}^{\infty} a_{l}(n) e^{-l u}=\pi \sum_{l=0}^{\infty} \alpha_{l} e^{-l u}
$$

Since the $a_{l}(n)$ are positive it follows that for each $l$ the sequence $\left\{a_{l}(n)\right\}$ is bounded. Thus by a familiar diagonal procedure a subsequence $\left\{n_{k}\right\}$ can be chosen so that

$$
\lim _{k \rightarrow \infty} a_{l}\left(n_{k}\right)=\beta_{l} .
$$

Furthermore (7.8) implies that

$$
\sum_{0}^{\infty} \beta_{l} e^{-l u}<\infty
$$

for every $u>0$ and hence

$$
\lim _{k \rightarrow \infty} \sum_{l=0}^{\infty} a_{l}\left(n_{k}\right) e^{-l u}=\sum_{l=0}^{\infty} \beta_{l} e^{-l u} .
$$

Thus (using (7.8) again)

$$
\beta_{l}=\pi \alpha_{l}, \quad l=0,1,2, \cdots
$$

and essentially the same argument shows that (7.9) can be replaced by the stronger statement

$$
\lim _{n \rightarrow \infty} a_{l}(n)=\beta_{l}=\pi \alpha_{l} .
$$

Thus (7.6a) and (7.6b) have been proved.

8. The discrete case. Similar results can be obtained if the $X$ 's have a lattice structure. The theory is actually much simpler and instead of the integral equation one obtains a finite set of linear equations.

Let the $X$ 's be such that

$$
\text { Prob. }\left\{X_{j}=k\right\}=c_{k}
$$

and we shall assume

$$
c_{k}=c_{-k}
$$

$\Omega$ is now a finite set of integers and 


$$
K_{z}(x)=\frac{1}{2 \pi} \int_{0}^{2 \pi} \frac{\cos \xi x}{1-z \phi(\xi)} d \xi,
$$

where $x$ is now an integer and

$$
\phi(\xi)=\sum_{-\infty}^{\infty} c_{k} e^{i k \xi}=c_{o}+2 \sum_{1}^{\infty} c_{k} \cos k \xi .
$$

We also have

$$
h(z)=\frac{1}{2 \pi} \int_{0}^{2 \pi} \frac{d \xi}{1-z \phi(\xi)}, \quad L(x)=\frac{1}{2 \pi} \int_{0}^{2 \pi} \frac{1-\cos \xi x}{1-z \phi(\xi)} d \xi .
$$

The analogue of the "backward" equation (3.5) is now

$$
S(\eta)=\frac{1}{1-z}-z\left(1-e^{-u}\right) \sum_{x \in \Omega} S(x) K_{z}(\eta-x)
$$

where

$$
S(0)=1+\sum_{1}^{\infty} z^{n} E\left\{\exp \left[-u \sum_{1}^{n} V\left(s_{k}\right)\right]\right\} .
$$

It is now easy to show that

$$
\lim _{z \rightarrow 1}(1-z) h(z) S(\eta)=G(u ; \eta)
$$

exists, as well as the limit

$$
\lim _{z \rightarrow \infty} h(z)\left(1-z h(z)\left(1-e^{-u}\right) \sum_{x \in \Omega} S(x)\right)=p(u) .
$$

One thus obtains

$$
G(u ; \eta)=p(u)+\left(1-e^{-u}\right) \sum_{x \in \Omega} G(u ; x) L(x-\eta)
$$

and

$$
\left(1-e^{-u}\right) \sum_{x \in \Omega} G(u ; x)=1 .
$$

Since $K_{z}(x-y)(x, y \in \Omega)$ is clearly a positive definite matrix one obtains as before that the matrix $L(x-y)(x, y \in \Omega)$ has exactly one positive eigenvalue if $\Omega$ contains more than one element. One obtains an amusing special case by considering $\phi(\xi)=\cos \xi$ (coin tossing). In this case

$$
L(x)=\frac{1}{2 \pi} \int_{0}^{2 x} \frac{1-\cos \xi x}{1-\cos \xi} d \xi=|x| \quad(x \text { an integer })
$$


and hence if $a_{1}<a_{2}<\cdots<a_{n}$ are integers (comprising the set $\Omega$ ) the matrix

$$
\left(\left(\left|a_{i}-a_{j}\right|\right)\right)
$$

has exactly one positive eigenvalue (if $n \geqq 2$ ). The remainder of the theory proceeds exactly as before. The reader will also note that the methods are applicable to a wide class of Markoff chains. For example, if $P_{n}(l ; \Omega)$ is the probability that an ordinary plane random walk (Pólya walk) will hit the finite set $\Omega$ of lattice points exactly $l$ times during the first $n$ steps the limit

$$
\lim _{n \rightarrow \infty} \log n P_{n}(l ; \Omega)
$$

exists and can be calculated by solving an appropriate set of linear equations.

One meets new problems and difficulties when one considers processes (continuous time). It is here that one is led to Potential Theory and these questions will be dealt with in a subsequent publication.

\section{REFERENCE}

1. D. A. Darling and M. Kac, On occupation times for Markoff processes, Trans. Amer. Math. Soc. vol. 84 (1957) pp. 444-458.

Cornell University, ITHACA, N. Y. 